\title{
Skin Detection and Segmentation of Human Face in Color Images
}

\author{
Baozhu Wang $^{1 *}$, Xiuying Chang ${ }^{2 *}$, Cuixiang Liu ${ }^{1}$ \\ ${ }^{1}$ Hebei University of Technology, Tianjin 300130, China \\ ${ }^{2}$ Cangzhou Vocational College of Technology, Cangzhou, China \\ *Corresponding authors'Email: wbz_china@126.com, changxiuyinglzzf@126.com
}

\begin{abstract}
This paper presents the procedures of face segmentation in the color image based on skin detection through the establishment of skin model and the segmentation of skin region. Firstly, in order to reduce the effect of factors on the segmentation of face region, a method for compensating the color of input images is used to alleviate the interferences from bad illuminating conditions. Secondly, Gaussian model about skin information is established which can be used to detect skin pixels in color images and transform color images to gray-scale images. Thirdly, a new algorithm of Otsu is used to find out the skin regions in binary images. Finally, mathematical morphology operator and prior knowledge are used to find out the face regions and discard regions that are similar to the skin in color. This method can deal with various sizes of faces, different illumination conditions, diverse poses and changeable expressions. In particular, the scheme significantly increases the execution speed of the face segmentation algorithm in the case of complex backgrounds. The experiments show that this method reduces the computation of the procedure, and at the same time improves the detection speed and efficiency.
\end{abstract}

Keywords: color balance; space conversion; Gaussian model; gray-scale image enhancement; adaptive threshold segmentation

\section{Introduction}

As one of human's physiological features, skin information has broad applications such as face detection, gesture analysis, target tracking and image retrieval. Skin has the advantage of being non-sensitive to directions, so we can separate skin regions from other parts of the color images and segment face regions accurately through post-processing. The application of color can provide valuable candidate region when detecting stationary targets. The combination of information from moving-targets can enhance the accuracy of target tracking and the analysis of property when detecting moving targets. According to the needs of search and retrieval system requirements, skin color can be used in image classification and feature analysis when retrieving image. Many researches of skin detection have been proposed. An example study of skin colors is Soriano's work [1] which built a skin color model in normalized color coordinates (NCC). Soriano established the model based on an extensive collection of skin color pixels on images of different races of people under a variety of illuminating conditions. Garcia and Tziritas [2] also built their skin color models over and HSV color spaces to extract skin pixels as candidate regions of faces. Bak [3] incorporated a 2D Gaussian skin model associated with motion information to find candidate faces. But most have many restrictions, such as no compensation for varying illumination colors, no noisy defocus, which hinder researchers from developing successful face detection systems. So, we present an improved procedure of skin detection and segmentation which can find out arbitrarily tilted human faces in color images. 
In this paper, we propose a skin-detection approach by using Gaussian model and skin-color information. Gaussian model about skin information is established to detect skin pixels in color images and transform color images to gray-scale images. Then a new algorithm of segmentation integrated histogram with Otsu is used to find out the skin regions in binary images. After that, mathematical morphology operator and prior knowledge are used to find out the face regions and discard regions that are similar to the skin in color. The rest of this paper is organized as follows. In Section 2, skin model is introduced. A skin segmentation algorithm is proposed in Section 3. In Section 4, experiments are conducted and discussed. Lastly, conclusions are given in Section 5.

\section{Skin Model}

For the segmentation of human faces based on skin color, the key is to select the color space and its cluster. In color images, skin color is very useful information for human face. Using skin-color information effectively can reduce the amount of searching time when it needs to make sure of the region of human face. However, skin-color information is often influenced by some factors. For instance, light environment and image acquisition equipment will lead to color offset. Before skin-color segmentation, we should do some pre-processing for images to alleviate the interferences from bad light. Taking account of the effects of light source and the high-brightness region in images on skin detection, we use the assumptive method of Gray World [4] in order to compensate the images with light interference. In addition, considering the brightness information and chrominance that should be separated, we segment the skin of color image in the space of $Y C_{b} C_{r}$ and set up Gaussian model which can cluster the skin-color information in a regular range $[5,6]$.

\subsection{Compensation for varying illumination col- ors}

In color images, skin color is relatively concentrated in a stable region. Skin-color information can be used to separate human faces from the background of images. Studies have shown that for different race, age and gender, face color looks different, but they mainly concentrate in brightness. Skin color coming from different human races has good clustering in the chrominance space. So, it is feasible to segment skin region based on this principle. But affected by the light source, image acquisition equipment and other factors, color images are often in color shift, so a light compensation preprocessing is proposed to alleviate the interferences from bad illuminating conditions.

Before light compensation preprocessing, we should determine whether there is light interference in color images. If not, we should not do light compensation preprocessing, or, it will lead to big change of skincolor information and affect the result of skin model. From the theory of dual-color reflection model [7], we know that the surface of objects appears the surface or interface reflection, so objects often send off brightlight. In color images, high-brightness region will be found. The surface reflection of objects mainly comes from the real color of light source when the light illuminates the objects, but not the color of the surface of objects. Based on these facts, the steps of determining whether the images have the light interference are as follows:

Step 1: The brightness of all the pixels in the image is put in order from top to low, taking the top 5\% of the pixels. If the number of pixels is more than enough (such as more than 1000), then calculate the respective average of these pixels component $(R, G, B)$ and record as $A R, A G, A B$;

Step 2: Calculate the value of $\max (A R, A G$, and $A B) /$ $\min (A R, A G$, and $A B)$. If the value greatly deviates from 1 , then determine the image has light interference.

If the image has light interference, the light compensation preprocessing of the Gray World is proposed to alleviate the interferences from bad illuminating conditions. The steps are as follows:

Step 1: Calculate their respective average and total mean of these components $(R, G$, and $B)$ and record as $\operatorname{avg} R, \operatorname{avg} G, \operatorname{avg} B, \operatorname{avg}$ Gray. The formula is as follow $: \operatorname{avg} G r a y=(\operatorname{avg} R+\operatorname{avg} G+\operatorname{avg} B) / 3$.

Step 2: Calculate the adjustment factors of $R, G$ and $B$, then record as $a R, a G$ and $a B$, the formulas are as

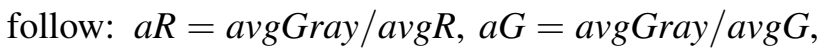
$a B=\operatorname{avgGray} / \operatorname{avg} B$.

Step 3: Adjust the values of $R, G$ and $B$ with the adjustment factors. The formulas are as follow: $R=$ $R a R ; G=G a G ; B=B a B$.

Step 4: If the value of $R, G$ and $B$ after adjustment is more than 255 , then adjust it to 255 .

Figure 1 shows the compensation result for tow original images. The color of image is reddish, whose skin color is very close to the color of the background, which will lead to the error of skin-color segmentation. 

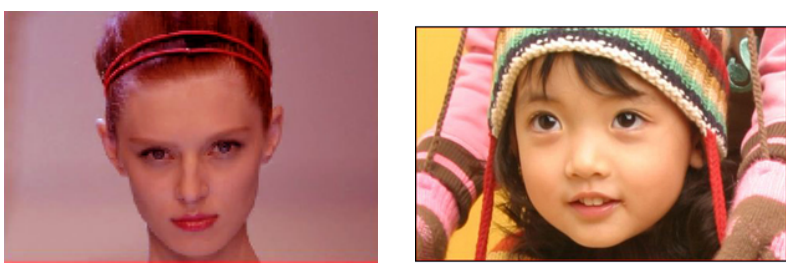

Figure 1 Original Images

Use the method which has been given in the previous part, we can alleviate the interferences from bad illuminating conditions in these images. Figure 2 shows the result of light compensation.
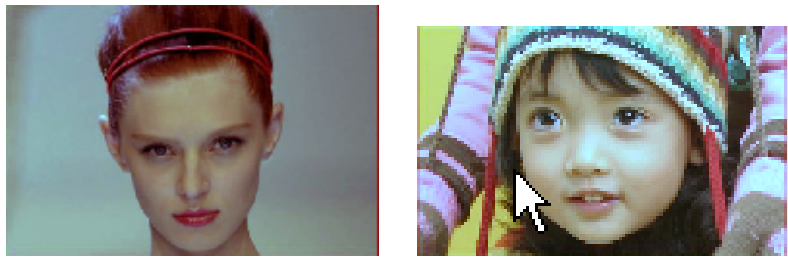

Figure 2 Light-compensated Images

\subsection{Changes in different color spaces}

In order to segment human skin regions from nonskin regions based on color, a reliable skin color model is needed that is adaptable to people of different skin colors and to different lighting conditions. The study has shown that the common $R G B$ representation of color images has three characters. First, two grayscale images which have the similar forms are likely under a huge difference in color space. Second, skincolor information coming from different people, different races can cluster in one color space. Last, luminance is the most important factor that affects the changes in different color spaces, but not skin color. Based on these natures, the color spaces that are frequently used in studies are $R G B$ (three primary colors), $r g b$, HIS, $Y C_{b} C_{r}$ (the chrominance model of CCIR601 encoding), UCS(CIE proposed a uniform color system) and $Y I Q$.

A color space is a mathematical expression of the color collection. The common $R G B$ representation of color images is not suitable for characterizing skincolor. In the $R G B$ space, the triple component $(r, g, b)$ represents not only color but also luminance. Luminance may vary across a person's face due to the ambient lighting and is not a reliable measure in separating skin from non-skin region. Luminance can be removed from the color representation in the chromatic color space. In this paper, we choose the $Y C_{b} C_{r}$ color space as the space of skin detection. $Y C_{b} C_{r}$ colors have been effectively used to segment color images in many applications. It is also well suited in this case to segment skin regions from non-skin regions. The color distribution of skin colors of different people was found to be clustered in a small area of the chromatic color space. Although skin colors of different people appear to vary over a wide range, they differ much less in color than in brightness. In other words, skin colors of different people are very close, but they differ mainly in intensities. With this finding, we could proceed to develop a skin-color model in the $Y C_{b} C_{r}$ color space. $Y C_{b} C_{r}$ color space can be transformed from $R G B$, and the corresponding coordinate transformation matrix are as follows:

$$
\left[\begin{array}{c}
Y \\
C_{b} \\
C_{r}
\end{array}\right]=\left[\begin{array}{ccc}
0.2990 & 0.5870 & 0.1140 \\
-0.1687 & -0.3313 & 0.5000 \\
0.5000 & -0.4187 & -0.0813
\end{array}\right]\left[\begin{array}{c}
R \\
G \\
B
\end{array}\right]
$$

Generally speaking, the result is much better when the luminance and color separate completely. Actually, there are certain of non-linear relations between them to some extent which will impact the image detection. So, it is needed to reduce these non-linear relations, and eliminating the relations between luminance and color. The transformation formulas that are used to change $Y C_{b} C_{r}$ color space to other space which has non-linear relations are as follows:

$$
C_{i}(Y)= \begin{cases}\left(C_{i}(Y)\right. & \left.-\overline{C_{i}(Y)}\right) \times \frac{W_{C_{i}}}{W_{C_{i}}(Y)}+\overline{C_{i}(Y)} \\ & \text { if }\left(Y<K_{i}\right) \operatorname{or}\left(K_{h}<Y\right) \\ C_{i}(Y) & \text { if }\left(Y \in\left[K_{i}, K_{h}\right]\right)\end{cases}
$$

where $i$ represents $b$ or $r$ ( $b$ and $r$ are the value of the $r g b$ image), $\overline{C_{i}(Y)}$ denotes the axis of skin-color regions, $K_{i}$ and $K_{h}$ are sub-threshold of non-linear transformation, $W_{C_{i}}(Y)$ is the width of the skin-color regions.

Figure 3 shows the images in $Y C_{b} C_{r}$ color space, which is different from the $R G B$ space.
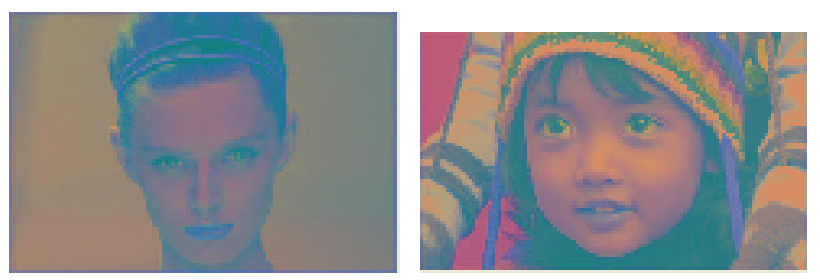

Figure 3 Images in $Y C_{b} C_{r}$ Color Space

\subsection{Gaussian model}

Although skin colors of different people appear to vary over a wide range, they are very close in color 


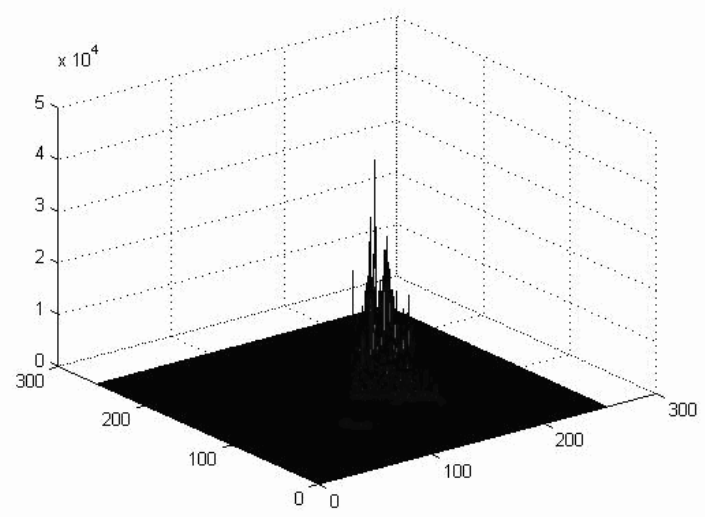

Figure 4 Skin Distribution in $Y C_{b} C_{r}$ Space

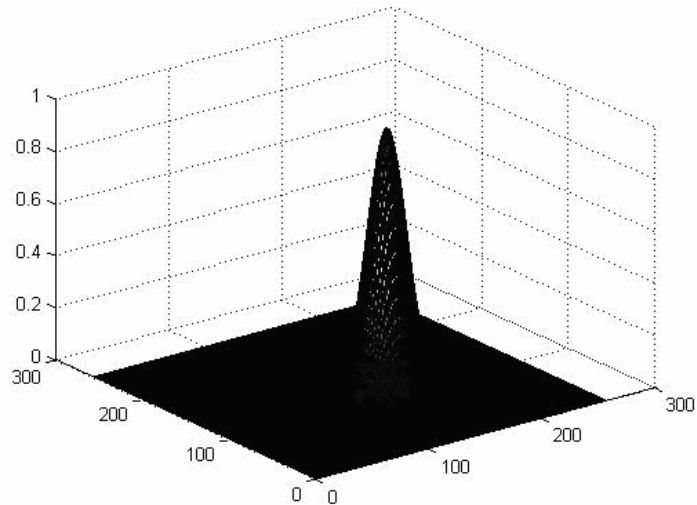

Figure 5 Gaussian Model and have huge differences in luminance. The color distribution of skin color of different people was found to be clustered in a small area of the color space and a skin color distribution can be represented by a Gaussian model.

According to the Gaussian distribution in the color space of images, a color image with skin-color regions can be transformed into a gray scale image so that the gray value at each pixel shows the likelihood of the pixel belonging to the skin. The likelihood of skin for this pixel can then be computed as follows:

$$
\begin{aligned}
& m=E\{x\} \\
& x=\left(C_{b}, C_{r}\right)^{T} \\
& C=E\left\{(x-m)(x-m)^{T}\right\} \\
& P\left(C_{b}, C_{r}\right)=\exp \left\{-0.5(x-m)^{T} C^{-1}(x-m)\right\}
\end{aligned}
$$

where $m$ is the mean of $x, C$ is the covariance matrix. We can obtain $m$ and $C$ through the sample statistics.

This skin model is based on the statistical characteristics [8], which needs to commutate the likelihood of each pixel belonging to the skin, so the speed is not fast. Of course, when doing the practical application of skin detection, you can directly use the formula of $-0.5(x-m)^{T} C^{-1}(x-m)$ in order to improve the detection speed. A total of 500 images of human faces were used to determine the color distribution of human skin in $Y C_{b} C_{r}$ color space. Our samples were taken from people of different ethnicities, different conditions and different ages. Figure 4 and Figure 5 have shown the statistical characteristics of skin information and Gaussian model.

Through Gaussian Model, the image in $Y C_{b} C_{r}$ Space can be transformed to a gray scale image which has the obvious skin regions. With appropriate threshold, the gray scale images can then be further transformed to a binary image showing skin regions and non-skin regions.

\section{Skin Segmentation}

Image segmentation is the key step of image processing to image analysis, and the foundation of identification and understanding, which mainly uses the differences between the target objects and its background segmenting the target regions which are wanted in need. There are many segmentation algorithms proposed by scholars, including threshold method, boundary detection, matching method and so on. In this paper, we use facial characteristics to separate the skincolor region from the images and get the binary image of human face regions, which is called threshold segmentation algorithm. This method can segment the wanted objects from background by choosing a suitable threshold, which is based on the different characteristics in gray-scale between the target and background. The most representative methods are histogram threshold, Otsu, the best entropy method, moment invariant method, fuzzy clustering method, a very small error of law and co-occurrence matrix method. With the advantages of small amount of calculation, simple theory and easy implementation, Otsu is generally used in real-time image processing system. Therefore, Otsu is used in this paper to segment the gray-scale image transformed from RGB in order to get the binary image. Then, process the binary image to obtain the accurate face region.

\subsection{The method of Otsu}

Otsu was proposed in Japan, which is derived on the principles of discrimination and the theory of least 
square.

There is a image with the gray-scale between one to $m$, the number of pixels with the value of $i$ is $n_{i}$, then the total number of image pixels is:

$$
N=\sum_{i=1}^{m} n_{i}
$$

The probability of appearing gray-scale pixel with the value of $i$ is:

$$
p_{i}=\frac{n_{i}}{N}
$$

Then, use $T$ to split the gray scale into two groups, $C_{0}=\{1 \sim T\}$ and $C_{1}=\{T+1 \sim m\}$. The formulas of the average and probability generated by each group are as follows:

$$
\left\{\begin{array}{c}
\omega_{0}=\sum_{i=1}^{T} p_{i}=\omega(T) \\
\mu_{0}=\sum_{i=1}^{T} \frac{i p}{\omega}=\frac{\mu(T)}{\omega(T)}
\end{array}\right.
$$

and

$$
\left\{\begin{array}{r}
\omega_{1}=\sum_{i=T+1}^{m} p=1-\omega \\
\mu_{1}=\sum_{i=T+1}^{m} \frac{i p}{\omega}=\frac{\mu-\mu(T)}{1-\omega(T)}
\end{array}\right.
$$

where $\mu=\sum_{i=1}^{m} i p_{i}$ is the formula for calculating the mean of the whole image, $\mu(T)=\sum_{i=1}^{T} i p_{i}$ is the formula for calculating the average of the image when the threshold value is $T$. So the formula of the gray sample average is $\mu=\omega_{0} \mu_{0}+\omega_{1} \mu_{1}$. The formula for calculating the variance between the two groups is as follows:

$$
\begin{aligned}
\delta^{2}(T) & =\omega_{0}\left(\mu_{0}-\mu\right)^{2}+\omega_{1}\left(\mu_{1}-\mu_{0}\right)^{2} \\
& =\omega_{0} \omega_{1}\left(\mu_{1}-\mu_{0}\right)^{2}=\frac{[\mu \omega(T)-\mu(T)]^{2}}{\omega(T)[1-\omega(T)]}
\end{aligned}
$$

Seek the maximum value of the formula, when $T$ is changing from one to $\mathrm{m}$. Then $T$ is the threshold and $\delta^{2}(T)$ is the selecting function of $\delta^{2}(T)$ [9].

\subsection{Improved segment method}

Based on the principle of threshold segmentation, we will segment the skin-likelihood images. With the original images affected by non-face regions, light, rotation angle of face and many other factors, the skinlikelihood image can't reflect good differences between background and the objects, which thus affect the results of a follow-up partition. Different from the previous procedure, this article does not directly deal with the similar gray-scale images, but first needs to determine whether the skin-likelihood images should be adjusted. This can improve the accuracy of segmentation to a large extent, although it will be affected by the factors such as light. Specific methods are as follows [10]:

Step 1: Get the histogram of the similar gray-scale image histogram, and determine the brightness and contrast of the image according to the distribution of pixel images in histogram. If the pixels are in the lowend of the histogram, they are illustrated in dark images; if the pixels are in the high-end of the histogram, they are illustrated in brightness images; if the pixels are in the center of the histogram, they are illustrated in the images with poor contrast; only when the gray histogram is more evenly distributed throughout the gray-scale range, is the image contrast relatively good.

Step 2: According to the above conditions, compare the histogram of the similar gray-scale images with the conditions one by one, then the treatment is necessary to enhance the needed image.

Step 3: Get the histogram of the new gray-scale image and set the value which is in the trough of the histogram as the threshold A. The gray space will be divided into two parts, part a and part b, by this threshold. Then get the variance of this two parts, and determine the values in the troughs of histograms in part $\mathrm{a}$ and part $\mathrm{b}$ separately. And make these two values as the boundaries which is the range of A moving, thus obtain the best threshold.

Experiments show that the improved method of segmentation not only accelerates the speed of segmentation, but also ameliorates the accuracy of skin segmentation. As shown in Figure 6 are the skin-likelihood image and the binary image.

\subsection{Determination of human faces}

However, it is important to note that the detected regions may not necessarily correspond to skin. It is only reliable to conclude that the detected regions have the same color as that of the skin. The important point here is that this process can reliably point out regions that do not have the color of the skin and such regions would not need to be considered anymore in the face finding process. In this paper, mathematical morphology operator of open computing is used to deal with the binary image [11]. Use the following methods to deal with the non-face region. 

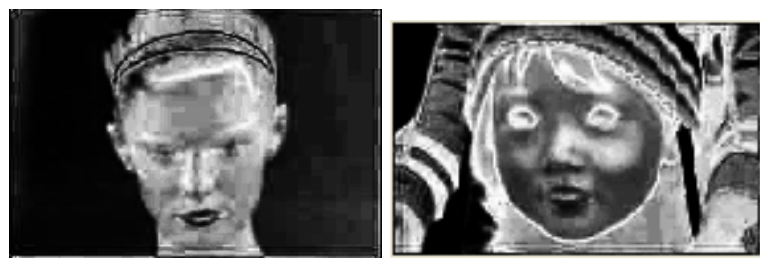

(a) Skin-likelihood Image

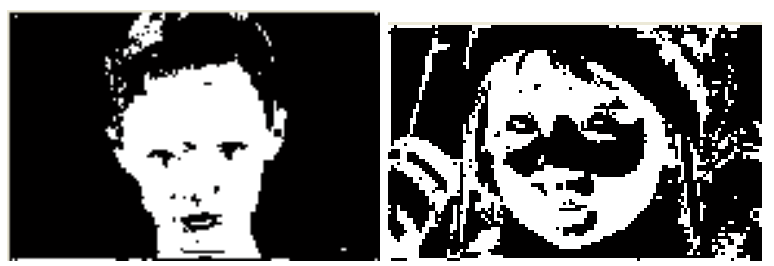

(b) Binary Image

Figure 6 Skin-likelihood Image and Binary Image

Step 1: Calculate the number of holes in each skin region, if there are no holes, and then discard the skin region. Use Euler's formula to calculate the number of holes.

Step 2: Find the width and the height in external rectangular of skin region, then compute the ratio of wide and high. After that, give up the skin region whose proportion is not demanded, and the range of ratio is between 0.8 with 2.0. If the value is more than 2.0 , use the lesser part of the ratio of 2.0 to match, and minimize the occurrence of undetected face, instead of discarding it [12].

Step 3: Calculate the ratio of skin area and its external rectangular, and discard the non-human face region with color characteristics; its ratio should be more than or equal to 0 [13].

Step 4: Remove the region whose area is less than 400. If the region of human faces is small, facial features will be lost during the pretreatment.

Step 5: Discard skin region whose width or height is less than 20 .

According to this method, most of the non-face region can be removed, which can segment face region accurately, as is shown in Figure 7.

\section{Results and Discussion}

In this paper, the software platform of matlab is used in face segmentation, which mainly targets the color images that are static. Considering there is no standard color-image database which can assess the faces in images, we set up the color-image database with 500 skin images. There are some samples of the images in Figure 8.

The pictures are from a variety of images, and have
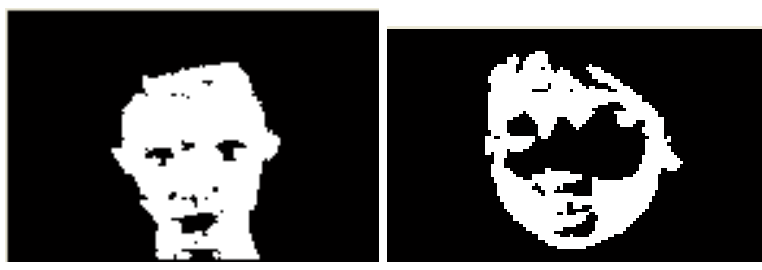

(a) Facial Region in Binary

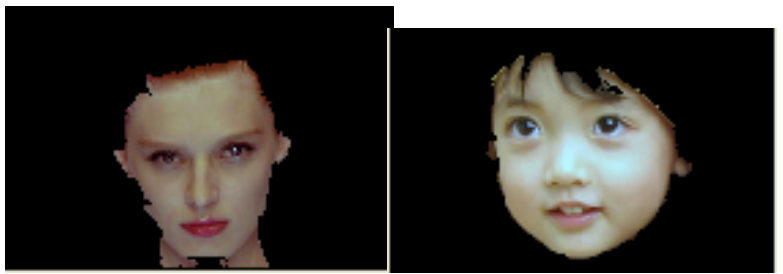

(b) Facial Skin Region

Figure 7 Final Results of Segmentation

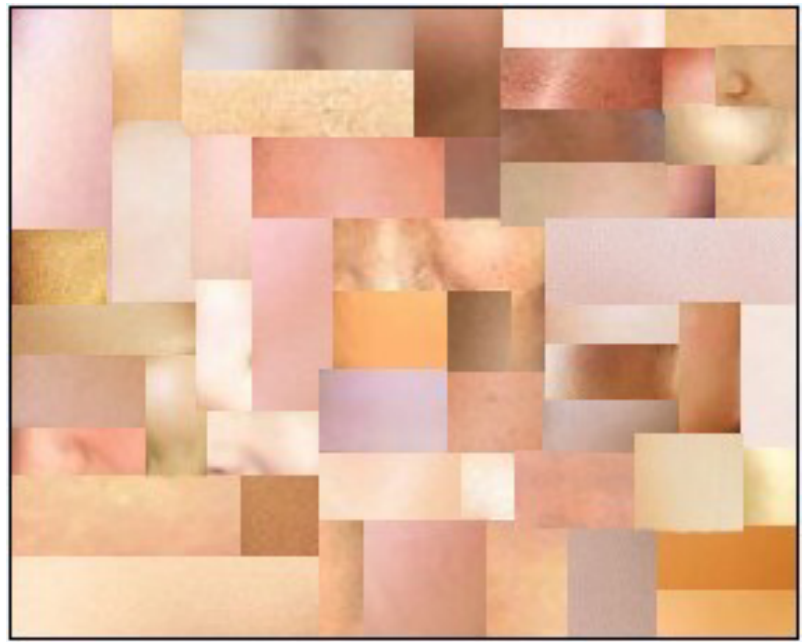

Figure 8 Samples of skin image

different colors and illumination, thereby meeting the requirements of skin model. With the skin model and the skin segmentation algorithm, we can deal with many images which include the images from the colorimage database and other images which come from internet and private camera. The effect of image segmentation on those two categories is basically same and good.

Experiments have shown that face region can be detected effectively and quickly based on the methods of this article. The results and discussion are as follows.

The original image of the experiment has been shown in Figure 1 which has light interference. But with the method of compensation for varying illumination color, the image has become normal skin color as is shown in Figure 2. And with the method of skin segmentation, we can get the first image of Figure 9(a).

Part $a$ and Part $b$ in Figure 9 show that whether or 

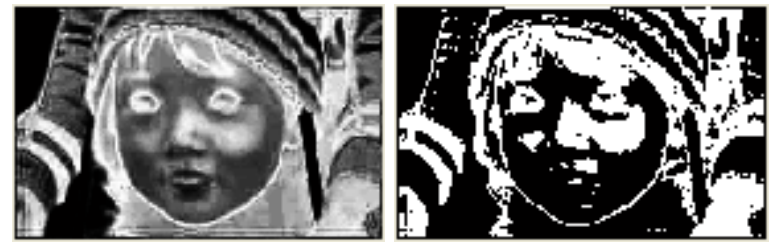

(a) Results with no color balance and enhancement
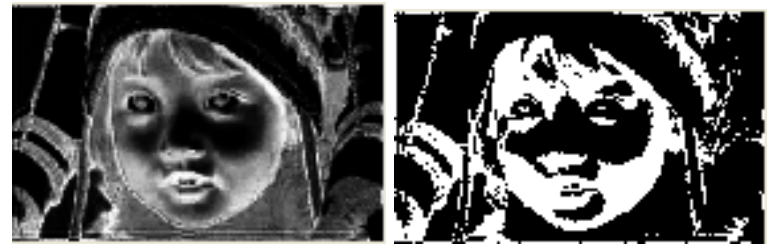

(b) Results with color balance and no enhancement

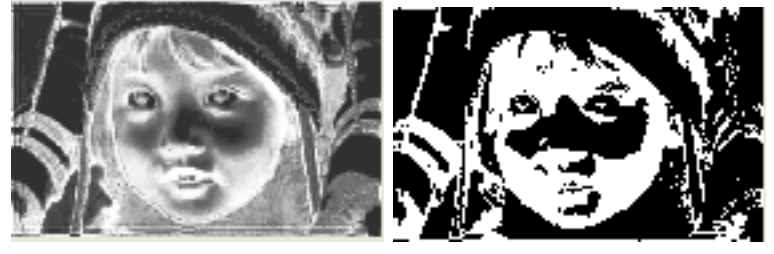

(c) Results with color balance and enhancement

Figure 9 Result of Image Comparison

Table 1 Statistical results of skin segmentation

\begin{tabular}{|c|c|}
\hline Classes & $\begin{array}{c}\text { Accuracy of } \\
\text { Segmentation } \%\end{array}$ \\
\hline no-color balance & 75.8 \\
\hline $\begin{array}{c}\text { color balance but no } \\
\text { image enhancement }\end{array}$ & 87.6 \\
\hline $\begin{array}{c}\text { color balance and } \\
\text { image enhancement }\end{array}$ & 94.3 \\
\hline
\end{tabular}

not to balance the image which has the light interference will directly affect the skin-likelihood image, causing that the face region can't be divided from the image. From part $b$ and part $c$, we can see that the enhancing process on the skin-likelihood image which mainly is reinforced through histogram equalization can improve the effect and accuracy of skin segmentation. This method can segment face regions better and there are the results of different images in Figure 10.

From Table 1, we can see that balancing the color image with light interference can improve the accuracy of segmentation, and the same as enhancing the skin-likelihood image with lower contrast. While, compared with ordinary Otsu, the improved Otsu will also accelerate the speed nearly twice, as shown in Table 2.
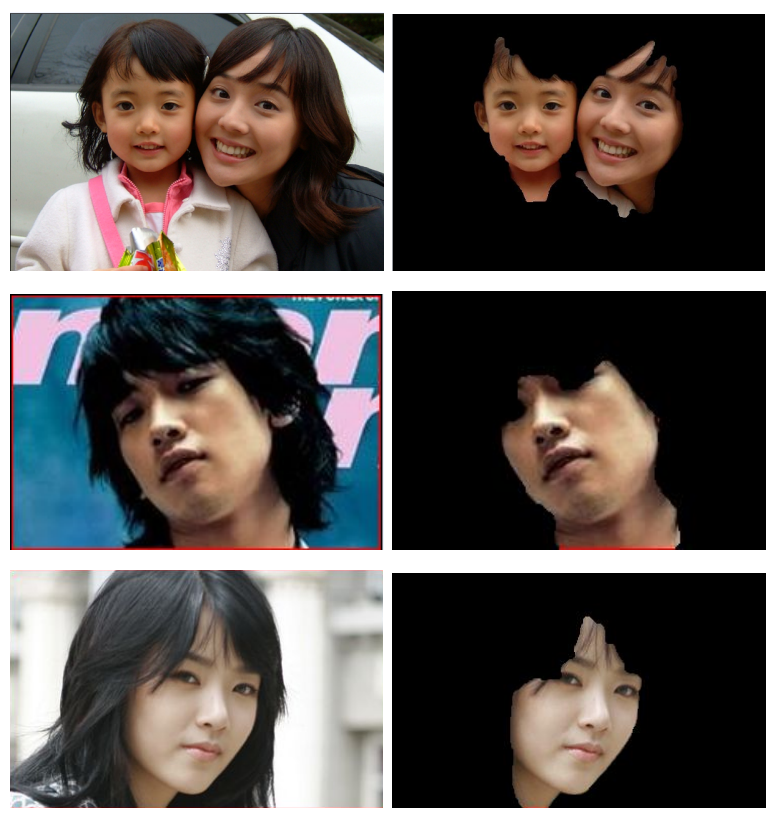

Figure 10 Results of face segmentation

Table 2 The speed of skin segmentation

\begin{tabular}{|c|c|}
\hline $\begin{array}{c}\text { Method of } \\
\text { Segmentation }\end{array}$ & $\begin{array}{c}\text { Time of } \\
\text { Segmentation/s }\end{array}$ \\
\hline Otsu & 4.4186 \\
\hline Improved Otsu & 2.4137 \\
\hline
\end{tabular}

\section{Conclusion}

With its complex and ever-changing nature, including the effect of the light and the condition of shooting environment, it makes the skin segmentation of human faces in color images severely affect face detection, and also makes it an important research topic. A method of face-region segmentation based on skin detection has been proposed in this paper, which partly comes from other studies. Compared with the conventional method of segmentation, we put these methods into this article, such as adjudging the images with the light interference, enhancing the images and improved threshold segmentation. Determination of the light interference not only improves the accuracy of image segmentation in the follow-up processing, but also expands the scope of application with skin segmentation in color images. Image enhancement mainly deals with the skin-likelihood image which is transformed through Gaussian model, aiming at getting the gray images with better and higher contrast. In this article, we use the method that combines the histogram with Otsu, which is the initial use of histogram threshold method to determine the threshold, and then we set it as the threshold of Otsu, and respectively, find the best 
threshold through searching the threshold to both ends which are in the limited scope. This method avoids the partition in the whole range of gray scale, thereby reducing the time of determining the optimum threshold, and provides real-time processing that guarantees the face detection system based on skin detection. Using these methods can handle various sizes of faces, different illumination conditions, diverse poses and changeable expressions. In particular, the scheme significantly increases the execution speed of the face segmentation algorithm in the case of complex backgrounds. In the future, the proposed method will be applied to face detection, face recognition and face tracking effectively.

\section{Acknowledgments}

The research is supported by Hebei University of Technology, China. The authors would like to thank Prof. Ya-tong Zhou for his proofreading of this paper. We also deeply appreciate the comments and suggestions of the anonymous reviewers who have helped us improve the quality of both the paper and our research.

\section{References}

[1] Soriano, M., Huovinen, S., "Using the Skin locus to cope with changing illumination conditions in colorbased face tracking", IEEE Nordic Signal Processing Symposiun, Kolmarden,Sweden. pp. 383-386, 2000.

[2] Garcia, C., Tziritas, G., "Face Decetion Using Quantized Skin color Regions Merging and Wavelet Packet Analysis", IEEE Transactions on Multimedia. pp. 264-277, 1999.

[3] Bark, S.Y., Lee, C.W., "PCA-base real-time face detection and tracking", International Technical Conference on Circuits, Computers and Communications. pp. 615-618, 2002.

[4] W. Jianguo, W. Jiangtao, Y. Jingyu, "Rotationinvariant Face Detection in Color Images with Complex Background", Computer Engineering, vol. 34, Feb. 2008, pp. 210-212.

[5] Q. Xiaofang, W. Xiaojun, W. Shitong, Yang Jingyu, "An improved algorithm of face detection", Computer Applications, vol. 28, Apr.2008, pp. 986-989.

[6] Chiunhsiun Lin. Face detection in complicated backgrounds and different illumination conditions by using $\mathrm{YCbCr}$ color space and neural network, Pattern Recognition Letters. 2007, 21902200

[7] Z. Shuzhen, S. Hailong, X. Xiaoyan, "Face Detection Based on Skin Segmentation and Features Location", Computer Engineering and Applications, vol. 14, Feb. 2008, pp. 8284
[8] Chen-Chin Chiang, Chi-Jang Huang, "A robust method for detecting arbitrarily tilted human faces in color images", Science, vol.26, Jul.2005, pp. 25182536, doi:10.1016/j.patrec.2005.05.008.

[9] C. Maoyuan, "Design of Color Image Skin Area Segmentation System in the Matlab Environment", Computer Applications, vol. 4,Nov. 2007, pp. 128-130.

[10] Q. Lina, Z. Bo, W. Zhanka, "Application of the OTSU Method inImage Processing", Radio engineering, vol.36, Jul. 2006, pp. 25-27.

[11] Hsiuao-Ying Chen, Chung-Lin Huang, Chih-Ming $\mathrm{Fu}$, "Hybird-boost learning for multi-pose face decetion and facial expression recognition", Pattern Recognition Society, vol. 41, Aug. 2007, pp. 11731185, doi:10.1016/j.patcog.2007.08.010.

[12] X. Mingkun,W. Houda, "A Method of Human Face Detection in Color Image Using Skin Color Segmentation", Electronic Engineer,vol.33, Mar. 2007, pp. $40-42$.

[13] S. Changyu, X. Panyuan, "Localization of human face based on skin module and skin color segmentation", Opto-Electronic Engineering, vol. 34, Sep. 2007, pp. 103-107 